\title{
An improved bound on the sizes of matchings guaranteeing a rainbow matching
}

\author{
Dennis Clemens Julia Ehrenmüller \\ Institut für Mathematik \\ Technische Universität Hamburg \\ Hamburg, Germany \\ \{dennis.clemens, julia.ehrenmueller\}@tuhh.de
}

Submitted: Mar 8, 2015; Accepted: Mar 30, 2016; Published: Apr 15, 2016

Mathematics Subject Classifications: 05C15, 05C70

\begin{abstract}
A conjecture by Aharoni and Berger states that every family of $n$ matchings of size $n+1$ in a bipartite multigraph contains a rainbow matching of size $n$. In this paper we prove that matching sizes of $\left(\frac{3}{2}+o(1)\right) n$ suffice to guarantee such a rainbow matching, which is asymptotically the same bound as the best-known one in the case where we only aim to find a rainbow matching of size $n-1$. This improves previous results by Aharoni, Charbit and Howard, and Kotlar and Ziv.
\end{abstract}

\section{Introduction}

In this paper we are concerned with the question which sizes of $n$ matchings in a bipartite multigraph suffice in order to guarantee a rainbow matching of size $n$.

One motivation for considering these kinds of problems is due to some well-known conjectures on Latin squares. A Latin square of order $n$ is an $n \times n$ matrix in which each symbol appears exactly once in every row and exactly once in every column. A partial transversal in a Latin square is a set of entries with distinct symbols such that from each row and each column at most one entry is contained in this set. We call a partial transversal of size $n$ in a Latin square of order $n$ simply a transversal. A famous conjecture of Ryser [11] states that for every odd integer $n$ any Latin square of order $n$ contains a transversal. The conjecture is known to be true for $n \leqslant 9$. Omitting the restriction to odd numbers yields a false statement. Brualdi $[7,8]$ and Stein [12] independently formulated the following conjecture for all orders $n$.

Conjecture 1 . For every $n \geqslant 1$ any Latin square of order $n$ has a partial transversal of size $n-1$. 
A natural way to transfer this problem to graphs is the following. Let $L=\left(\ell_{i, j}\right)_{i, j \in[n]}$ be a Latin square of order $n$. We define $G_{L}:=(A \cup B, E)$ as the complete bipartite edge-coloured graph with partite sets $A=\left\{a_{1}, \ldots, a_{n}\right\}$ and $B=\left\{b_{1}, \ldots, b_{n}\right\}$, where $a_{i} b_{j}$ is coloured $\ell_{i, j}$. That is, $A$ and $B$ represent the columns and rows of $L$, respectively. Moreover, a transversal of $L$ corresponds to a perfect matching in $G_{L}$ that uses each edge colour exactly once, which we call a rainbow matching of size $n$. Using this notion, Conjecture 1 is equivalent to the following: For every $n \geqslant 1$ any complete bipartite edgecoloured graph, the colour classes of which are perfect matchings, contains a rainbow matching of size $n-1$.

One may wonder whether this might even be true in the more general setting of bipartite edge-coloured multigraphs: Following Aharoni, Charbit and Howard [2], we define $f(n)$ to be the smallest integer $m$ such that every bipartite edge-coloured multigraph with exactly $n$ colour classes, each being a matching of size at least $m$, contains a rainbow matching of size $n$. Aharoni and Berger [1] conjectured the following generalization of Conjecture 1.

Conjecture 2. For every $n \geqslant 1$ we have $f(n)=n+1$.

The first approaches towards this conjecture are given by the bounds $f(n) \leqslant\left\lfloor\frac{7}{4} n\right\rfloor$ due to Aharoni, Charbit and Howard [2] and $f(n) \leqslant\left\lfloor\frac{5}{3} n\right\rfloor$ due to Kotlar and Ziv [10]. Here, we give an improved bound, which is asymptotically the same as the best-known bound on the sizes of the colour classes in the case where we aim to find a rainbow matchings of size $n-1$ [10]. In particular, we prove the following.

Theorem 3. For every $\varepsilon>0$ there exists an integer $n_{0} \geqslant 1$ such that for every $n \geqslant n_{0}$ we have $f(n) \leqslant\left(\frac{3}{2}+\varepsilon\right) n$.

Subsequently, we use the following notation. Let $G$ be a bipartite multigraph with partite sets $A$ and $B$. For sets $X \subseteq A$ and $Y \subseteq B$ we write $E_{G}[X, Y]$ to denote the set of edges in $G$ between $X$ and $Y$. Given some matching $R$ in $G, N_{G}(X \mid R):=\{y \in B$ : $\exists x y \in R$ with $x \in X\}$ denotes the neighbourhood of $X$ with respect to $R$. For the sake of readability, we omit floor and ceiling signs and do not intend to optimize constants in the proofs.

\section{Proof of Theorem 3}

In this section we give a proof of Theorem 3 the idea of which can be summarized as follows. We start with assuming for a contradiction that a maximum rainbow matching in the given graph $G=(A \cup B, E)$ is of size $n-1$. A rainbow matching of this size is known to exist [10]. We fix such a matching $R$ and find two sequences $e_{1}, \ldots, e_{k}$ and $g_{1}, \ldots, g_{k}$ of edges, the first consisting of edges from $R$ and the second consisting of edges outside $R$. We then show that either we can switch some of the edges between the edge sequences to produce a rainbow matching of size $n$ (see the proofs of the Claims 4, 5 and 7), or the matchings represented by the edges $e_{1}, \ldots, e_{k}$ need to touch at least $n$ vertices in $B$ 
that are saturated by $R$, both leading to a contradiction. To make the second case more precise we additionally introduce certain sequences $X_{1}, \ldots, X_{k} \subseteq A$ and $Y_{1}, \ldots, Y_{k} \subseteq B$ in the proof.

Proof. Let $\varepsilon>0$ be given and whenever necessary we may assume that $n$ is large enough. Let $\mathcal{F}=\left\{F_{0}, F_{1}, \ldots, F_{n-1}\right\}$ be a family of $n$ matchings of size at least $(3 / 2+\varepsilon) n$ in a bipartite multigraph $G=(A \cup B, E)$ with partite sets $A$ and $B$. We aim to find a rainbow matching of size $n$.

For a contradiction, let us assume that there is no such matching. As shown in [10], there must exist a rainbow matching $R$ of size $n-1$. We may assume without loss of generality that none of the edges of $F_{0}$ appears in $R$. Let $t$ be the smallest positive integer with $1 /(2 t-1) \leqslant \varepsilon$. Moreover, let $X \subseteq A$ and $Y \subseteq B$ be the sets of vertices that are saturated by $R$, i.e. incident with some edge of $R$.

In the following we show that for every $k \in[t]$ we can construct sequences

(S1) $e_{1}, \ldots, e_{k}$ of $k$ distinct edges $e_{i}=x_{i} y_{i}$ in $R$ with $x_{i} \in X$ and $y_{i} \in Y$,

(S2) $g_{1}, \ldots, g_{k}$ of $k$ distinct edges $g_{i}=z_{i} y_{i}$ with $z_{i} \in A \backslash X$,

(S3) $X_{1}, \ldots, X_{k}$ of subsets of $X$,

(S4) $Y_{1}=N_{G}\left(X_{1} \mid R\right), \ldots, Y_{k}=N_{G}\left(X_{k} \mid R\right)$ of subsets of $Y$,

and an injective function $\pi:\{0,1, \ldots, k\} \rightarrow\{0,1, \ldots, n-1\}$ with $\pi(0):=0$ such that the following properties hold:

(P1) for each $i \in[k]$ we have $e_{i} \in F_{\pi(i)}$,

(P2) for each $i \in[k]$ we have $g_{i} \in \bigcup_{j=0}^{i-1} F_{\pi(j)}$,

(P3) $\left(e_{1} \cup \cdots \cup e_{k}\right) \cap\left(X_{k} \cup Y_{k}\right)=\varnothing$,

(P4) $\left|X_{k}\right|=\left|Y_{k}\right|=s_{k}:=2 k \varepsilon n+k(7-3 k) / 2$,

(P5) for each $i \in[k]$ and each $j \in\{0, \ldots, n-1\}$ it holds that if $R$ contains an edge of the matching $F_{j}$ between $X_{i}$ and $Y_{i}$, then there is also an edge of $F_{j}$ between $x_{i}$ and $B \backslash Y$,

(P6) for each $i \in[k]$ and each $w \in Y_{i} \backslash Y_{i-1}$ there exists a vertex $v \in A \backslash\left(X \cup\left\{z_{1}, \ldots, z_{i-1}\right\}\right)$ such that $v w \in F_{\pi(i-1)}$ (where $\left.Y_{0}:=\varnothing\right)$, and

(P7) for each $i \in[k]$ and each $j \in[i-1]$ it holds that if $g_{i} \in F_{\pi(j)}$, then $z_{i} \in A \backslash(X \cup$ $\left.\left\{z_{1}, \ldots, z_{j}\right\}\right)$.

Let us explain some of the ideas behind Properties (P1)-(P7). Let $i \in[k]$, then Properties (P1), (P2) and (P7) ensure subsequences of the sequences in (S1) and (S2) such that switching between them produces another rainbow matching of size $n-1$ where the matching $F_{\pi(i)}$ is not present. Properties (P5) and (P6) guarantee a large number of 
switchings that produce different rainbow matchings of size $n-1$. This will help us to prove restrictions on where the edges of $F_{\pi(i)}$ can occur in the multigraph. In particular we show that $F_{\pi(i)}$ cannot have edges between some parts of the graph and the set $Y_{i}$ from (S4), the size of which increases with $i$.

Before we start with the construction, let us first observe that by Property (P4) we have a set $Y_{t} \subseteq Y$ which satisfies $2 t \varepsilon n+t(7-3 t) / 2=\left|Y_{t}\right| \leqslant|Y|<n$. However, for large enough $n$ and by the choice of $t$ we have that $2 t \varepsilon n+t(7-3 t) / 2>n$, a contradiction.

In order to find the sequences described above, we proceed by induction on $k$. For the base case, let us argue why we find edges $e_{1}, g_{1}$, sets $X_{1}, Y_{1}$, and an injective function $\pi$ with Properties (P1)-(P7). First observe that $F_{0}$ does not have any edges between $A \backslash X$ and $B \backslash Y$, by assumption on $R$. As $\left|F_{0}\right| \geqslant(3 / 2+\varepsilon) n$, there are at least $(1 / 2+\varepsilon) n+1$ edges of $F_{0}$ between $A \backslash X$ and $Y$. Let $N_{0} \subseteq Y$ denote a set of size $(1 / 2+\varepsilon) n+1$ such that for every vertex $w \in N_{0}$ there exists a vertex $v \in A \backslash X$ such that $v w \in F_{0}$. Furthermore, let $X_{1}^{\prime}:=N_{G}\left(N_{0} \mid R\right)$ and let $\mathcal{R}_{1}:=\left\{F_{j} \in \mathcal{F}: F_{j} \cap R \cap E_{G}\left[X_{1}^{\prime}, N_{0}\right] \neq \varnothing\right\}$, i.e. $\mathcal{R}_{1}$ consists of all matchings that have an edge in $R$ between $X_{1}^{\prime}$ and $N_{0}$.

As a next step we show that there exists an edge $e_{1}=x_{1} y_{1} \in R \cap E_{G}\left[X_{1}^{\prime}, N_{0}\right]$ such that there are $2 \varepsilon n+2$ matchings in $\mathcal{R}_{1}$ that have an edge between $x_{1}$ and $B \backslash Y$. The sets $X_{1} \subseteq X$ and $Y_{1}=N_{G}\left(X_{1} \mid R\right)$ will then be defined as the vertices that are incident with edges in $R$ that are contained in these $2 \varepsilon n+2$ matchings.

Let $F$ be any matching in $\mathcal{R}_{1}$, let $v w$ be the unique edge in $F \cap R \cap E_{G}\left[X_{1}^{\prime}, N_{0}\right]$ and let $z \in A \backslash X$ be the unique vertex such that $z w \in F_{0}$. Notice that there cannot be any edge $g$ of $F$ between $A \backslash(X \cup\{z\})$ and $B \backslash Y$, since otherwise $(R \backslash\{v w\}) \cup\{z w, g\}$ would give a rainbow matching of size $n$, in contradiction with $R$ being a maximum rainbow matching. Therefore, there are at least $(1 / 2+\varepsilon) n+1$ edges of $F$ between $B \backslash Y$ and $X \cup\{z\}$. Since $\left|X_{1}^{\prime}\right|=(1 / 2+\varepsilon) n+1$, there are at least $2 \varepsilon n+2$ edges of $F$ between $B \backslash Y$ and $X_{1}^{\prime}$. Since this is true for any $F \in \mathcal{R}_{1}$, we know by the pigeonhole principle that there is a vertex $x_{1} \in X_{1}^{\prime}$ and a subset $X_{1} \subseteq X_{1}^{\prime}$ of size $2 \varepsilon n+2$ such that, for every $F_{j} \in \mathcal{F}$, if $F_{j} \cap R \cap E_{G}\left[X_{1}, B\right] \neq \varnothing$, then $F_{j}$ has an edge between $x_{1}$ and $B \backslash Y$. Note that $x_{1} \notin X_{1}$. Let $e_{1}=x_{1} y_{1}$ be the unique edge in $R$ incident with $x_{1}$ and let $g_{1}=z_{1} y_{1}$ be the unique edge of $F_{0}$ incident with $y_{1} \in N_{0}$. Set $\pi(1)$ to be the unique index $j \in[k]$ such that $e_{1} \in F_{j}$. One can easily verify that $e_{1}=x_{1} y_{1}, g_{1}=z_{1} y_{1}, X_{1}, Y_{1}:=N_{G}\left(X_{1} \mid R\right)$, and $\pi$ satisfy Properties (P1)-(P7).

For the inductive step let us assume that for some $k \in[t-1]$ the above sequences are given with Properties (P1)-(P7). We now aim to extend these by edges $e_{k+1}, g_{k+1}$, sets $X_{k+1}, Y_{k+1}$, and a value $\pi(k+1)$ while maintaining Properties (P1)-(P7). The approach that we use is similar to the base case. We start with some useful claims that will guarantee us in Corollary 6 that the matching $F_{\pi(k)}$ has $(1 / 2+\varepsilon) n+1-2 k$ edges between $A \backslash\left(X \cup\left\{z_{1}, \ldots, z_{k}\right\}\right)$ and $Y \backslash\left(Y_{k} \cup\left\{y_{1}, \ldots, y_{k}\right\}\right)$.

Claim 4. $F_{\pi(k)}$ has no edge between $A \backslash\left(X \cup\left\{z_{1}, \ldots, z_{k}\right\}\right)$ and $B \backslash Y$.

Proof of Claim 4. Assume for a contradiction that there exists an edge $g \in F_{\pi(k)}$ between the sets $A \backslash\left(X \cup\left\{z_{1}, \ldots, z_{k}\right\}\right)$ and $B \backslash Y$. (See Figure 1 for an illustration.) By 
Property (P2) we find a sequence $k>j_{1}>j_{2}>\cdots>j_{s}=0$ with $1 \leqslant s \leqslant k$ such that

$$
\begin{aligned}
g_{k} & \in F_{\pi\left(j_{1}\right)}, \\
g_{j_{i}} & \in F_{\pi\left(j_{i+1}\right)} \text { for } i<s .
\end{aligned}
$$

Moreover, according to Property (P7) we know that $z_{k}, z_{j_{1}}, \ldots, z_{j_{s-1}}$ are distinct, and thus, also using Property (P1), we conclude that

$$
\left(R \backslash\left\{e_{k}, e_{j_{1}}, \ldots, e_{j_{s-1}}\right\}\right) \cup\left\{g_{k}, g_{j_{1}}, \ldots, g_{j_{s-1}}, g\right\}
$$

forms a rainbow matching which is larger than $R$, a contradiction.

A

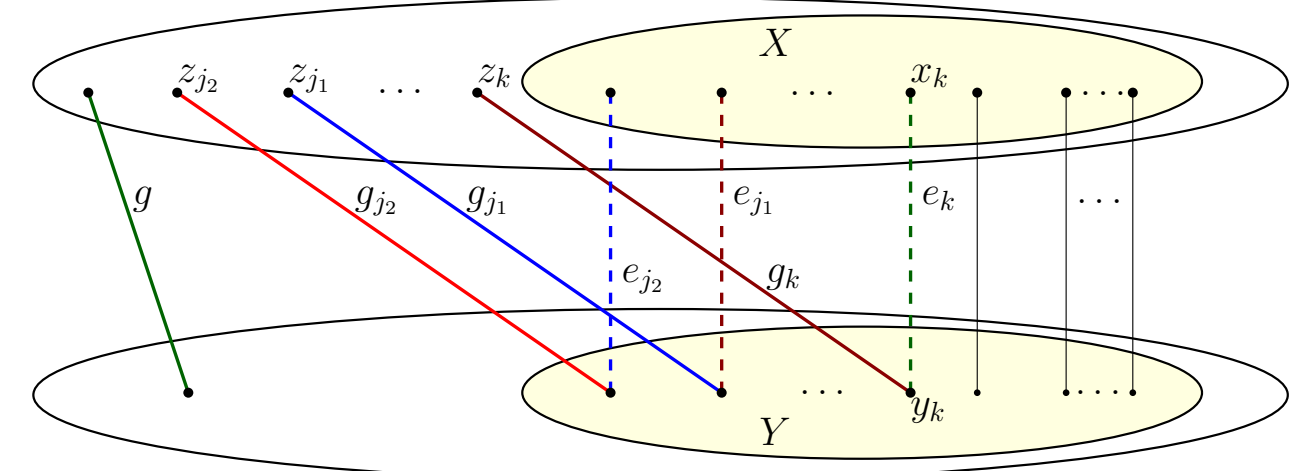

Figure 1: Example with $g_{j_{2}} \in F_{\pi(0)}(s=3)$. The dotted edges $\left\{e_{k}, e_{j_{1}}, e_{j_{2}}\right\}$ are replaced by the edges $\left\{g_{k}, g_{j_{1}}, g_{j_{2}}, g\right\}$ to obtain a larger rainbow matching.

Claim 5. $F_{\pi(k)}$ has no edge between $A \backslash\left(X \cup\left\{z_{1}, \ldots, z_{k}\right\}\right)$ and $Y_{k}$.

Proof of Claim 5. Assume for a contradiction that there is an edge $g \in F_{\pi(k)}$ between the sets $A \backslash\left(X \cup\left\{z_{1}, \ldots, z_{k}\right\}\right)$ and $Y_{k}$. (See Figure 2 for an illustration.) Let $e$ be the unique edge in $R$ which is adjacent to $g$. Observe that $e$ lies between $X_{k}$ and $Y_{k}$ by assumption. Let $j \in[n-1]$ be such that $e \in F_{j}$. By Property (P3) we have $e \notin\left\{e_{1}, \ldots, e_{k}\right\}$. Thus, using Property $(\mathrm{P} 1)$ and the fact that $R$ is a rainbow matching, we can conclude that $j \notin\{\pi(i): 1 \leqslant i \leqslant k\}$. Now, by Property (P5) it holds that there is an edge $\bar{e} \in F_{j}$ between $x_{k}$ and $B \backslash Y$. Moreover, by Properties (P2) and (P7), we find a sequence $k>j_{1}>j_{2}>\cdots>j_{s}=0$ with $1 \leqslant s \leqslant k$ such that

$$
\begin{aligned}
g_{k} & \in F_{\pi\left(j_{1}\right)}, \\
g_{j_{i}} & \in F_{\pi\left(j_{i+1}\right)} \text { for } i<s
\end{aligned}
$$

and all vertices $z_{k}, z_{j_{1}}, \ldots, z_{j_{s-1}}$ are distinct. Therefore, using Property (P1), we conclude that

$$
\left(R \backslash\left\{e_{k}, e_{j_{1}}, \ldots, e_{j_{s-1}}, e\right\}\right) \cup\left\{g_{k}, g_{j_{1}}, \ldots, g_{j_{s-1}}, \bar{e}, g\right\}
$$

forms a rainbow matching which is larger than $R$, a contradiction. 
A

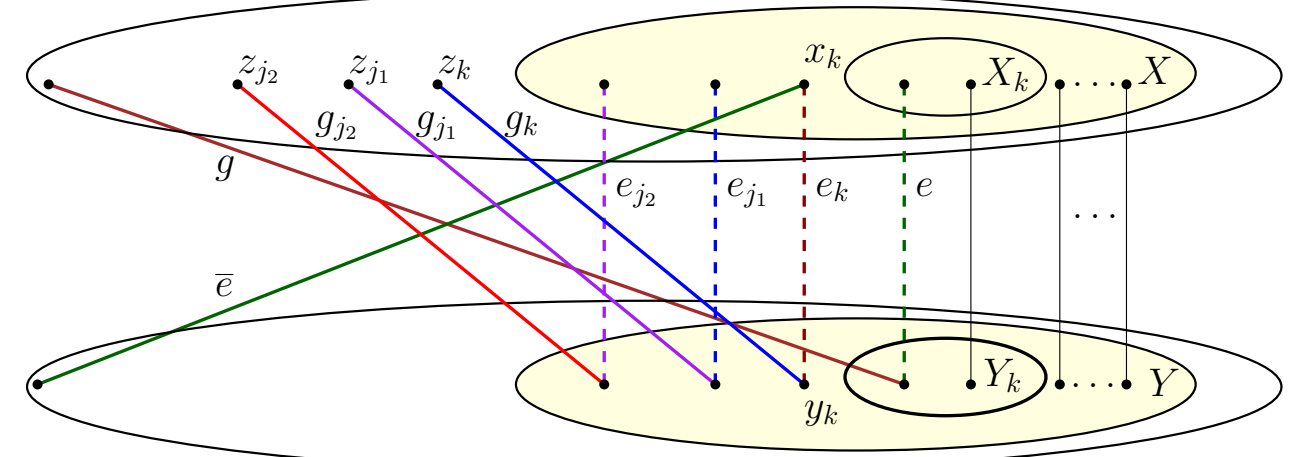

Figure 2: Example with $g_{j_{2}} \in F_{\pi(0)}(s=3)$. The dotted edges $\left\{e_{k}, e_{j_{1}}, e_{j_{2}}, e\right\}$ are replaced by the edges $\left\{g_{k}, g_{j_{1}}, g_{j_{2}}, \bar{e}, g\right\}$ to obtain a larger rainbow matching.

Corollary 6. The matching $F_{\pi(k)}$ has at least $\left(\frac{1}{2}+\varepsilon\right) n+1-2 k$ edges between $A \backslash\left(X \cup\left\{z_{1}, \ldots, z_{k}\right\}\right)$ and $Y \backslash\left(Y_{k} \cup\left\{y_{1}, \ldots, y_{k}\right\}\right)$.

Proof of Corollary 6. As $\left|F_{\pi(k)}\right| \geqslant(3 / 2+\varepsilon) n$ and $\left|X \cup\left\{z_{1}, \ldots, z_{k}\right\}\right| \leqslant n-1+k$, we conclude that at least $(1 / 2+\varepsilon) n+1-k$ edges of $F_{\pi(k)}$ are incident with vertices in $A \backslash\left(X \cup\left\{z_{1}, \ldots, z_{k}\right\}\right)$. Each of these edges intersects $Y \backslash Y_{k}$ by the previous claims and thus the statement follows.

In the following, let $N_{k} \subseteq Y \backslash\left(Y_{k} \cup\left\{y_{1}, \ldots, y_{k}\right\}\right)$ be a set of size $(1 / 2+\varepsilon) n+1-2 k$ such that for each vertex $w \in N_{k}$ there is a vertex $v \in A \backslash\left(X \cup\left\{z_{1}, \ldots, z_{k}\right\}\right)$ with $v w \in F_{\pi(k)}$. Such a set exists by the previous corollary. Moreover, let

$$
Y_{k+1}^{\prime}:=Y_{k} \cup N_{k}
$$

and let $X_{k+1}^{\prime}:=N_{G}\left(Y_{k+1}^{\prime} \mid R\right)$ be the neighbourhood of $Y_{k+1}^{\prime}$ with respect to $R$. By Property (P4), and as $N_{k} \cap Y_{k}=\varnothing$, we obtain

$$
\begin{aligned}
\left|X_{k+1}^{\prime}\right|=\left|Y_{k+1}^{\prime}\right| & =2 k \varepsilon n+\frac{k(7-3 k)}{2}+\left(\frac{1}{2}+\varepsilon\right) n+1-2 k \\
& =\frac{1}{2} n+(2 k+1) \varepsilon n+\frac{-3 k^{2}+3 k+2}{2} .
\end{aligned}
$$

We now look at all matchings that have an edge in $R$ between $X_{k+1}^{\prime}$ and $Y_{k+1}^{\prime}$. Formally, we consider

$$
\mathcal{R}_{k+1}:=\left\{F_{j} \in \mathcal{F}: F_{j} \cap R \cap E_{G}\left[X_{k+1}^{\prime}, Y_{k+1}^{\prime}\right] \neq \varnothing\right\} .
$$

Similarly to the base case we now aim to show that there exists an edge $e_{k+1}=$ $x_{k+1} y_{k+1} \in R \cap E_{G}\left[X_{k+1}^{\prime}, Y_{k+1}^{\prime}\right]$ such that there are $s_{k+1}$ matchings in $\mathcal{R}_{k+1}$ that have an edge between $x_{k+1}$ and $B \backslash Y$. This will follow immediately from the following claim and is stated in Corollary 8.

Claim 7. Every $F_{j} \in \mathcal{R}_{k+1}$ has at least $s_{k+1}$ edges between $X_{k+1}^{\prime}$ and $B \backslash Y$. 
Proof of Claim \%. The main argument is similar to that of Claim 4 - Corollary 6. For $F_{j} \in \mathcal{R}_{k+1}$ let $f=v w$, with $v \in X_{k+1}^{\prime}, w \in Y_{k+1}^{\prime}$, denote the unique edge in $F_{j} \cap$ $R \cap E_{G}\left[X_{k+1}^{\prime}, Y_{k+1}^{\prime}\right]$. Since $Y_{k+1}^{\prime}:=Y_{k} \cup N_{k}$, we either have $w \in Y_{k}$ or $w \in N_{k}$. In particular, by Property (P3) from the hypothesis and by the definition of $N_{k}$, we know that $w \notin\left\{y_{1}, \ldots, y_{k}\right\}$, and therefore $j \notin\{\pi(i): 0 \leqslant i \leqslant k\}$.

If $w \in Y_{k}$, then we find an integer $j_{1} \in[k]$ such that $w \in Y_{j_{1}} \backslash Y_{j_{1}-1}$ (where $Y_{0}:=\varnothing$ ), and by Property (P6) there is a vertex $z \in A \backslash\left(X \cup\left\{z_{1}, \ldots, z_{j_{1}-1}\right\}\right)$ such that $z w \in F_{\pi\left(j_{1}-1\right)}$. If otherwise $w \in N_{k}$, then we find a vertex $z \in A \backslash\left(X \cup\left\{z_{1}, \ldots, z_{k}\right\}\right)$ such that $z w \in F_{\pi(k)}$, by construction of $N_{k}$. In either case, let us fix this particular vertex $z$. We now prove the claim by showing first that (i) $F_{j}$ has no edge between $A \backslash\left(X \cup\left\{z_{1}, \ldots, z_{k}, z\right\}\right)$ and $B \backslash Y$, and then we conclude that (ii) the statement holds for $F_{j}$.

We start with the discussion of (i). So, assume for a contradiction that $F_{j}$ has an edge $\bar{f}$ between $A \backslash\left(X \cup\left\{z_{1}, \ldots, z_{k}, z\right\}\right)$ and $B \backslash Y$.

If $w \in Y_{k}$, then by the definition of $z$ we have $z w \in F_{\pi\left(j_{1}-1\right)}$, with $j_{1}$ being defined above. We can assume that $j_{1}>1$, as otherwise $z w \in F_{0}$ and thus $(R \backslash\{f\}) \cup\{\bar{f}, z w\}$ forms a full rainbow matching, in contradiction to our main assumption. Now, using Property (P2), we find a sequence $j_{1}-1>j_{2}>\cdots>j_{s}=0$ with $2 \leqslant s<k$ such that

$$
\begin{aligned}
g_{j_{1}-1} & \in F_{\pi\left(j_{2}\right)}, \\
g_{j_{i}} & \in F_{\pi\left(j_{i+1}\right)} \text { for } 2 \leqslant i \leqslant s-1
\end{aligned}
$$

By Property (P7) and since $z \in A \backslash\left(X \cup\left\{z_{1}, \ldots, z_{j_{1}-1}\right\}\right)$, the vertices $z, z_{j_{1}-1}, z_{j_{2}}, \ldots, z_{j_{s-1}}$ are distinct. We thus find the rainbow matching

$$
\left(R \backslash\left\{e_{j_{1}-1}, e_{j_{2}}, \ldots, e_{j_{s-1}}, f\right\}\right) \cup\left\{g_{j_{1}-1}, g_{j_{2}}, \ldots, g_{j_{s-1}}, \bar{f}, z w\right\}
$$

which is larger than $R$, a contradiction.

A

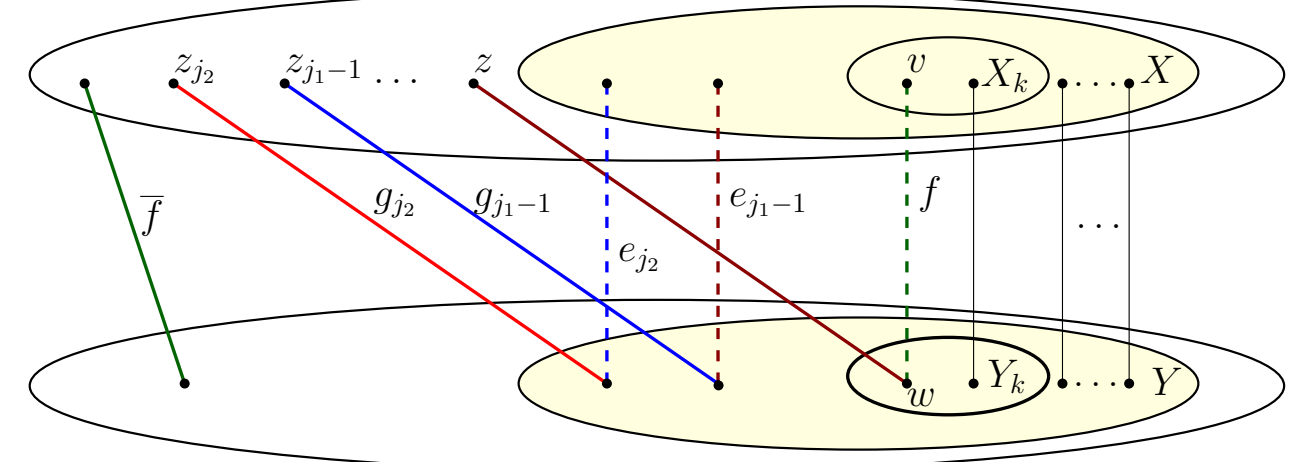

Figure 3: Example with $g_{j_{2}} \in F_{\pi(0)}$, in case $w \in Y_{k}$. The dotted edges $\left\{e_{j_{1}-1}, e_{j_{2}}, f\right\}$ are replaced by the edges $\left\{g_{j_{1}-1}, g_{j_{2}}, \bar{f}, z w\right\}$ to obtain a larger rainbow matching.

If otherwise $w \in N_{k}$, then $z w \in F_{\pi(k)}$. Analogously we find a sequence $k>j_{1}>j_{2}>$ $\cdots>j_{s}=0$ with $1 \leqslant s \leqslant k$ such that $g_{k} \in F_{\pi\left(j_{1}\right)}$ and $g_{j_{i}} \in F_{\pi\left(j_{i+1}\right)}$ for $i<s$, and we 
obtain a contradiction as

$$
\left(R \backslash\left\{e_{k}, e_{j_{1}}, \ldots, e_{j_{s}}, f\right\}\right) \cup\left\{g_{k}, g_{j_{1}}, \ldots, g_{j_{s}}, \bar{f}, z w\right\}
$$

forms a rainbow matching which is larger than $R$. Thus, we are done with part (i).

Let us proceed with (ii): $F_{j}$ needs to saturate at least $(1 / 2+\varepsilon) n+1$ vertices of $B \backslash Y$, as $\left|F_{j}\right| \geqslant(3 / 2+\varepsilon) n$ and $|Y| \leqslant n-1$. Thus, by part (i), we have at least $(1 / 2+\varepsilon) n+1$ edges of $F_{j}$ between $X \cup\left\{z_{1}, \ldots, z_{k}, z\right\}$ and $B \backslash Y$. Using $(*)$, we further calculate that

$$
\begin{aligned}
\left|X \cup\left\{z_{1}, \ldots, z_{k}, z\right\}\right|-\left|X_{k+1}^{\prime}\right| & \leqslant(n+k)-\left(\frac{1}{2} n+(2 k+1) \varepsilon n+\frac{-3 k^{2}+3 k+2}{2}\right) \\
& =\frac{1}{2} n-(2 k+1) \varepsilon n+\frac{3 k^{2}-k-2}{2} .
\end{aligned}
$$

Thus, the number of edges in $F_{j}$ between $X_{k+1}^{\prime}$ and $B \backslash Y$ needs to be at least

$$
\left(\frac{1}{2}+\varepsilon\right) n+1-\left(\frac{1}{2} n-(2 k+1) \varepsilon n+\frac{3 k^{2}-k-2}{2}\right)=s_{k+1},
$$

as claimed.

We now proceed with the construction of the edges $e_{k+1}, g_{k+1}$ and the sets $X_{k+1}, Y_{k+1}$, and afterwards we show that all required properties are maintained. The next corollary is by the pigeonhole principle an immediate consequence of Claim 7 .

Corollary 8. There exists a vertex $x_{k+1} \in X_{k+1}^{\prime}$, a set $X_{k+1} \subseteq X_{k+1}^{\prime}$ of size $s_{k+1}$ and its neighborhood $Y_{k+1} \subseteq Y_{k+1}^{\prime}$ with respect to $R$ such that the following holds for every $j \in[n-1]:$ If $F_{j} \cap R \cap E_{G}\left[X_{k+1}, Y_{k+1}\right] \neq \varnothing$, then $F_{j}$ has an edge between $x_{k+1}$ and $B \backslash Y$.

To extend the sequences, choose $X_{k+1}$ and $Y_{k+1}$ according to Corollary 8 , and let $e_{k+1}=x_{k+1} y_{k+1}$ be the unique edge in $R$ that is incident with $x_{k+1}$. Note that $x_{k+1} \notin$ $X_{k+1}$, as otherwise $x_{k+1}$ would need to be incident to two edges of the same matching $F_{j}$.

Observe that $y_{k+1} \notin\left\{y_{1}, \ldots, y_{k}\right\}$. Indeed, $y_{k+1} \in Y_{k+1}^{\prime}=Y_{k} \cup N_{k}$, and by construction we have $N_{k} \cap\left\{y_{1}, \ldots, y_{k}\right\}=\varnothing$, while $Y_{k} \cap\left\{y_{1}, \ldots, y_{k}\right\}=\varnothing$ holds by Property (P3).

Now, let $e_{k+1} \in F_{j}$. As $e_{k+1} \in R \backslash\left\{e_{1}, \ldots, e_{k}\right\}$, we have $j \notin\{\pi(i): 0 \leqslant i \leqslant k\}$. We extend the injective function $\pi$ with $\pi(k+1)=j$.

Finally, we choose $g_{k+1}$ as follows: If $y_{k+1} \in N_{k}$, then by construction of $N_{k}$ there is a vertex $z_{k+1} \in A \backslash\left(X \cup\left\{z_{1}, \ldots, z_{k}\right\}\right)$ with $z_{k+1} y_{k+1} \in F_{\pi(k)}$. Otherwise, if $y_{k+1} \in Y_{k}$, then there is an $i \in[k]$ with $y_{k+1} \in Y_{i} \backslash Y_{i-1}$, and by Property (P6) there is a vertex $z_{k+1} \in$ $A \backslash\left(X \cup\left\{z_{1}, \ldots, z_{i-1}\right\}\right)$ such that $z_{k+1} y_{k+1} \in F_{\pi(i-1)}$. In any case, we set $g_{k+1}:=z_{k+1} y_{k+1}$.

Claim 9. The extended sequences satisfy Properties (P1)-(P7).

Proof of Claim 9. Properties (P1) and (P2) follow immediately from the induction hypothesis and from the definition of $\pi(k+1)$ and $g_{k+1}$. By construction, we have $Y_{k+1} \subseteq$ $Y_{k+1}^{\prime}=Y_{k} \cup N_{k}$. By Property (P3) of the induction hypothesis and by the definition of 
$N_{k}$, we have $\left\{y_{1}, \ldots, y_{k}\right\} \cap Y_{k+1}=\varnothing$. It follows from the construction of $X_{k+1}$ (Corollary 8) that $y_{k+1} \notin Y_{k+1}$. By symmetry, we have $\left\{e_{1}, \ldots, e_{k+1}\right\} \cap\left(X_{k+1} \cup Y_{k+1}\right)=\varnothing$, which shows Property (P3). Properties (P4) and (P5) hold by Corollary 8 and by Property (P5) of the induction hypothesis. Recall that $Y_{k+1} \backslash Y_{k} \subseteq N_{k}$. This means that for every $w \in Y_{k+1} \backslash Y_{k}$ there exists a vertex $v \in A \backslash\left(X \cup\left\{z_{1}, \ldots, z_{k}\right\}\right)$ such that $v w \in F_{\pi(k)}$, proving Property (P6). Finally, Property (P7) holds by the induction hypothesis and since we chose $z_{k+1}$ from a set $A \backslash\left(X \cup\left\{z_{1}, \ldots, z_{i-1}\right\}\right)$ such that $z_{k+1} y_{k+1} \in F_{\pi(i-1)}$ for the appropriate $i \in[k+1]$. Consequently, all Properties (P1)-(P7) are fulfilled by the extended sequences.

Claim 9 concludes the induction and thus the proof of Theorem 3.

\section{Open problems and concluding remarks}

In this paper we proved that a collection of $n$ matchings of size $(3 / 2+o(1)) n$ in a bipartite multigraph guarantees a rainbow matching of size $n$. For smaller matching sizes it is even unknown whether a rainbow matching of size $n-1$ exists. More generally, as suggested by Tibor Szabó (private communication), it would be interesting to determine upper bounds on the smallest integer $\mu(n, \ell)$ such that every family of $n$ matchings of size $\mu(n, \ell)$ in a bipartite multigraph guarantees a rainbow matching of size $n-\ell$. One can verify that $\mu(n, l) \leqslant \frac{l+2}{l+1} n$. Moreover, it holds that $\mu(n, \sqrt{n}) \leqslant n$, which is a generalization (see e.g. [3]) of a result proved in the context of Latin squares by Woolbright [13], and independently by Brouwer, de Vries and Wieringa [6].

In order to approach Conjecture 2, one can also increase the number of matchings and fix their sizes to be equal to $n$ instead of considering families of $n$ matchings of sizes greater than $n$. Drisko [9] proved that a collection of $2 n-1$ matchings of size $n$ in a bipartite multigraph with partite sets of size $n$ guarantees a rainbow matching of size $n$. He also showed that this result is sharp. This problem was investigated in the following two directions. Does the statement also hold if we omit the restriction on the sizes of the vertex classes? And how many matchings do we need to find a rainbow matching of size $n-\ell$ for every $\ell \geqslant 1$ ? Aharoni and Berger [1] affirmed the first question by showing that for any integers $s \leqslant t$, the maximal number of matchings of size $t$ in a bipartite multigraph that do not contain a rainbow matching of size $s$ is equal to $2(s-1)$. The second question was studied recently by Barát, Gyárfás and Sárközy in [4]. They proved that for every $\ell \geqslant 1$ any bipartite multigraph with $\left\lfloor\frac{\ell+2}{\ell+1} n\right\rfloor-(\ell+1)$ matchings of size $n$ has a rainbow matching of size $n-\ell$. This result is sharp for $\ell=0$ and $\lfloor n / 2\rfloor \leqslant \ell<n$. For $\ell=\lfloor\sqrt{n}\rfloor$ it extends the result $\mu(n, \sqrt{n}) \leqslant n$ to the setting of multigraphs.

Finally, in case Conjecture 2 turns out to be true, it is of interest to see how sharp it is. As shown by Barat and Wanless [5], one can find constructions of $n$ matchings with $\left\lfloor\frac{n}{2}\right\rfloor-1$ matchings of size $n+1$ and the remaining ones being of size $n$ such that there is no rainbow matching of size $n$. We wonder whether the expression $\left\lfloor\frac{n}{2}\right\rfloor-1$ above could also be replaced by $(1-o(1)) n$. 


\section{References}

[1] R. Aharoni and E. Berger. Rainbow matchings in r-partite r-graphs. Electronic Journal of Combinatorics, 16(1):R119, 2009.

[2] R. Aharoni, P. Charbit, and D. Howard. On a Generalization of the Ryser-BrualdiStein Conjecture. Journal of Graph Theory, 78(2):143-156, 2015.

[3] R. Aharoni, D. Kotlar, R. Ziv. Rainbow sets in the intersection of two matroids. Electronic Notes in Discrete Mathematics, 43:39-42, 2013.

[4] J. Barát, A. Gyárfás, and G. N. Sárközy. Rainbow matchings in bipartite multigraphs. arXiv: 1505.01779.

[5] J. Barát and I. M. Wanless. Rainbow matchings and transversals. Australasian Journal of Combinatorics, 59(1):211-217, 2014.

[6] A. E. Brouwer, A. J. de Vries, and R. M. A. Wieringa. A lower bound for the length of partial transversals in a Latin square. Nieuw Archief Voor Wiskunde, 26:330-332, 1978.

[7] R. A. Brualdi and H. J. Ryser. Combinatorial matrix theory. Cambridge University Press, 1991.

[8] J. Dénes and A. D. Keedwell. Latin squares and their applications. Academic Press, New York, 1974.

[9] A. A. Drisko. Transversals in row-Latin rectangles. Journal of Combinatorial Theory, Series A, 84(2):181-195, 1998.

[10] D. Kotlar and R. Ziv. Large matchings in bipartite graphs have a rainbow matching. European Journal of Combinatorics, 38:97-101, 2014.

[11] H. J. Ryser. Neuere Probleme der Kombinatorik. Vorträge über Kombinatorik, Oberwolfach, pages 69-91, 1967.

[12] S. K. Stein. Transversals of Latin squares and their generalizations. Pacific Journal of Mathematics, 59(2):567-575, 1975.

[13] D. E. Woolbright. An $n \times n$ Latin square has a transversal with at least $n-\sqrt{n}$ distinct symbols. Journal of Combinatorial Theory, Series A, 24(2):235-237, 1978. 